\title{
Vergleich subjektiver und automatisierter Merkmalsextraktion sowie Einsatz maschineller Lernalgorithmen zur mikromagnetischen Materialcharakterisierung
}

\author{
Sargon Youssef ${ }^{1}$, Cyril Zimmer ${ }^{1}$, Klaus Szielasko ${ }^{1}$, Zeeshan Khan Suri ${ }^{1}$, Andreas Schütze ${ }^{2}$ \\ ${ }^{1}$ Fraunhofer Institut für Zerstörungsfreie Prüfverfahren IZFP \\ Campus E3 1 \\ 66123 Saarbrücken, Deutschland \\ ${ }^{2}$ Lehrstuhl für Messtechnik, Universität des Saarlandes \\ Gebäude A5 1 \\ 66123 Saarbrücken, Deutschland
}

\begin{abstract}
Zusammenfassung
Im Rahmen dieses Beitrags wurde untersucht, mit welchen Möglichkeiten die datenbasierte Vorhersagequalität verbessert werden kann. Neben der Optimierung des Merkmalsraums wurde die multiple lineare Regression (ML-R) mit weiteren Regressionsalgorithmen (Support Vector Regression, Multilayer Perceptron Regression) verglichen. Diese Regressoren bieten aufgrund ihrer Mächtigkeit und Flexibilität, Nichtlinearitäten in den Daten abzubilden, enormes Potenzial zur Erstellung komplexer Modelle. Beide besitzen jedoch "Stellschrauben“ (sog. Hyperparameter) die signifikant zur Vorhersagequalität beitragen und aufgrund der Vielzahl möglicher Kombinationen kann das Finden einer (quasi-) optimalen Lösung sehr aufwendig sein. Zur Verdeutlichung dieses Aufwandes wurden im Experiment alle Hyperparameter bis auf einen je Regressor vorgegeben. Dieser diente als Stellgröße und sollte aufzeigen, welcher Aufwand schon bei der Variation einzelner Hyperparametern zum Finden eines (quasi-) optimalen Modells erforderlich sind.
\end{abstract}

Keywords: Zerstörungsfreie Prüfung, Elektromagnetische Prüfverfahren, Materialcharakterisierung, Maschinelles Lernen, Regression

\section{Einleitung}

Änderungen mechanischer Kenngrößen wie beispielsweise Härte, Zugfestigkeit oder Streckgrenze, aber auch Last- und Eigenspannungen korrelieren mit Änderungen von Merkmalen der magnetischen Hysteresekurve ferromagnetischer Werkstoffe (z. B. Koerzitivfeldstärke, Remanenz, Permeabilität, etc.) [1]. Diese gegenseitige Abhängigkeit im Werkstoffverhalten erlaubt die Bestimmung mechanischer Kenngrößen auf Basis magnetischer Hystereseeigenschaften, die alternativ teilzerstörend bzw. zerstörend ermittelt werden (z. B. Vickershärte, metallografische Gefügeuntersuchungen, röntgenographische Bestimmung des Eigenspannungszustandes, etc.). Die direkte Bestimmung der magnetischen Hysterese an Bauteilen und Halbzeugen ist häufig in Aufsatztechnik prüfgerecht nicht möglich, teilweise aufgrund der hohen Messzeit, teilweise aufgrund der Notwendigkeit, wohldefiniert geformte Proben zu entnehmen, um die Flussdichte über eine probenumfassende Spule zu messen [1].

\section{MA-X8 Prüfverfahren}

Das am Fraunhofer-Institut für Zerstörungsfreie Prüfverfahren IZFP entwickelte 3MA-X8Verfahren (3MA $=$ Mikromagnetische Multiparameter Mikrostruktur und SpannungsAnalyse) kombiniert in Echtzeit drei Prüfmethoden in Aufsatztechnik mit unterschiedlicher Eindringtiefe und Sensitivität und extrahiert hieraus indirekte Hysteresemerkmale, um auch im Hinblick auf Störeinflüsse zur Zielgröße (z. B. Einsatz verschiedener Werkstoffe, überlagerte Last- und Eigenspannung, etc.) eine zuverlässige Charakterisierung des Werkstoffzustandes zu ermöglichen [2]. Hierbei wird ein Elektromagnet spannungsgesteuert zur Erregung eines magnetischen Wechselfeldes eingesetzt ( $\mathrm{vgl}$. Abb. 1). 


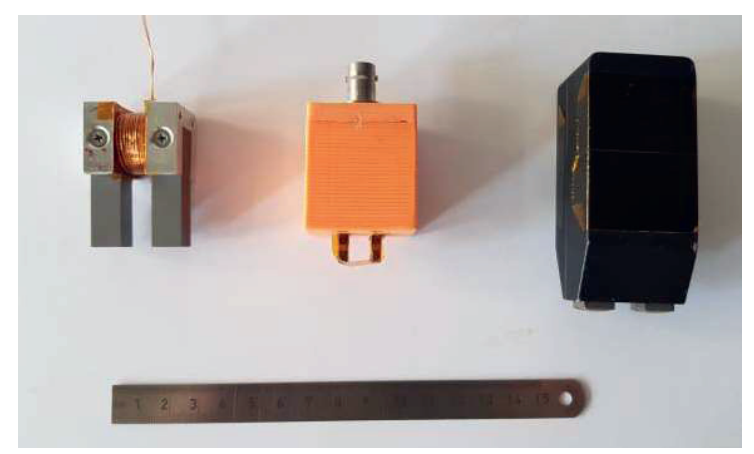

Abb.1: Unterschiedliche Elektromagnete als 3MA-X8 Prüfkopf

Dieses magnetische Wechselfeld setzt sich zusammen aus einem niederfrequenten Anteil $(10 \mathrm{~Hz}-200 \mathrm{~Hz})$ und einem höherfrequenten Anteil $(500 \mathrm{~Hz}-5000 \mathrm{~Hz})$ und wird bei Aufsetzen des Elektromagneten auf einen Werkstoff durch dessen Permeabilität und Leitfähigkeit beeinflusst. Diese Abhängigkeit zeigt sich unter anderem im Realteil $\operatorname{Re}(Z)$ und Imaginärteil $\operatorname{Im}(Z)$ der Impedanz und im Spulenstrom des Elektromagneten Ic [2]

\section{Subjektive Merkmalsextraktion 3MA-X8}

Basierend auf den zur Verfügung stehenden zeitlichen Signale können charakteristische, aber subjektive Merkmale aus den Messkurven extrahiert werden (z. B. Phasenverschiebung des Spulenstroms unten in Abb. 2) [2]. Insgesamt steht ein mehrdimensionaler Merkmalsraum mit insgesamt 21 Merkmalen zur Verfügung.

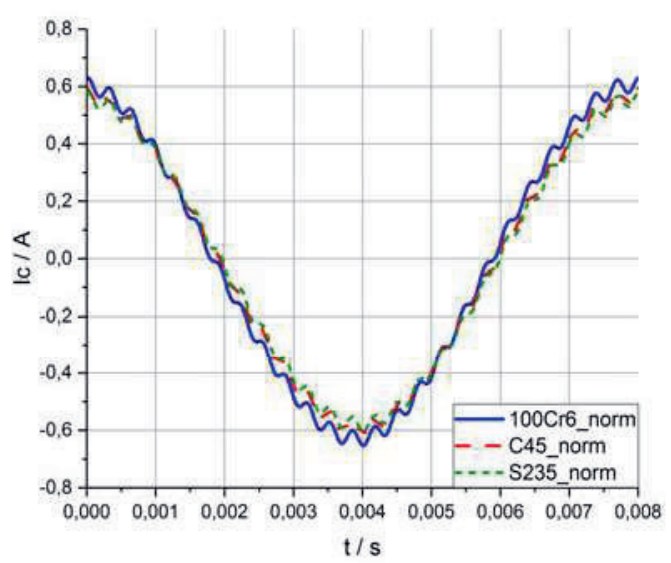

Abb.2: Spulenstrom für drei verschiedene normalisierte Werkstoffe (100Cr6, C45, S235)

Bei dieser Merkmalsextraktion besteht die Möglichkeit, dass geringe, aber signifikante Messeffekte in den Messkurven übersehen werden und der mikromagnetische Merkmals- raum das mechanische Werkstoffverhalten nicht bestmöglich abbildet.

\section{Automatisierte Merkmalsextraktion 3MA-X8}

Eine automatisierte Merkmalsextraktion bietet die Möglichkeit, Merkmale auch mit kleinem Messeffekt zur Zielgröße systematisch und unabhängig vom Beobachter zu extrahieren und dadurch die Vorhersagequalität (engl. Performance) von Werkstoffeigenschaften zu verbessern [2].

Bei der automatisierten Merkmalsextraktion werden die zur Verfügung stehenden zeitlichen Signale genauer analysiert. Beispielsweise wird der Spulenstrom mit Hilfe einer FourierTransformation in seine Frequenzanteile zerlegt und Betrag und Phase der unterschiedlichen Oberwellen als Merkmale extrahiert [2].

\section{Experiment}

In Abhängigkeit der Aufgabenstellung (Klassifizierung bzw. Quantifizierung von Werkstoffen und Werkstoffeigenschaften) werden die extrahierten Merkmale unter Verwendung maschineller Lernalgorithmen zur zerstörungsfreien Materialcharakterisierung eingesetzt. Kleinere Einflüsse auf die magnetische Hysterese (z. B. durch Zugspannungen) lassen sich ebenfalls bestimmen. Bei überlagerten Einflüssen, wie der gleichzeitigen Verwendung verschiedener Werkstoffe verschlechtert sich jedoch die Vorhersagequalität aufgrund von Mehrdeutigkeiten der Daten im Merkmalsraum. Im Vergleich zum ursprünglich 21-dimensionalen Merkmalsraum liefert die automatisierte Merkmalsextraktion mit über 270 Merkmalen einen höherdimensionaleren Merkmalsraum. Zur Beurteilung der Qualität des Merkmalsraum ist jedoch nicht die Anzahl der Merkmale relevant, sondern der Informationsgehalt in und zwischen diesen. Zum Vergleich und zur Bewertung wurde ein Experiment durchgeführt, in der zylinderförmige Proben dreier gehärteter und anschließend angelassener Werkstoffe (100Cr6, C45, S235) in eine Zugmaschine eingespannt und mit elf unterschiedlichen Zugspannungen (von 0 bis $50 \%$ der Streckgrenze $\mathrm{Re}_{\mathrm{e}}$ des Werkstoffs mit einer Schrittweite von $5 \% R_{e}$ ) belastet wurden (vgl. Abb. 3). 


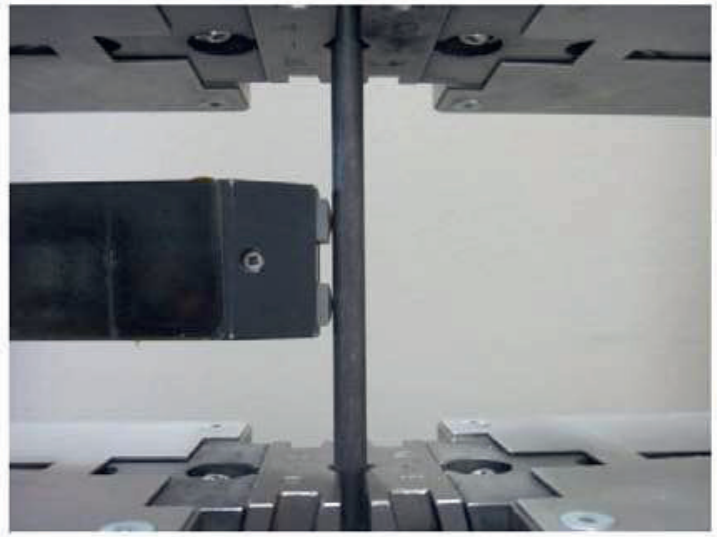

Abb.3: Eingespannte Probe mit aufgesetztem 3MA-X8 Prüfkopf

Im Anschluss wurde auf Basis der beiden Merkmalsräume eine multiple lineare Regressionsanalyse (ML-R) zur Vorhersage des Spannungszustandes durchgeführt.
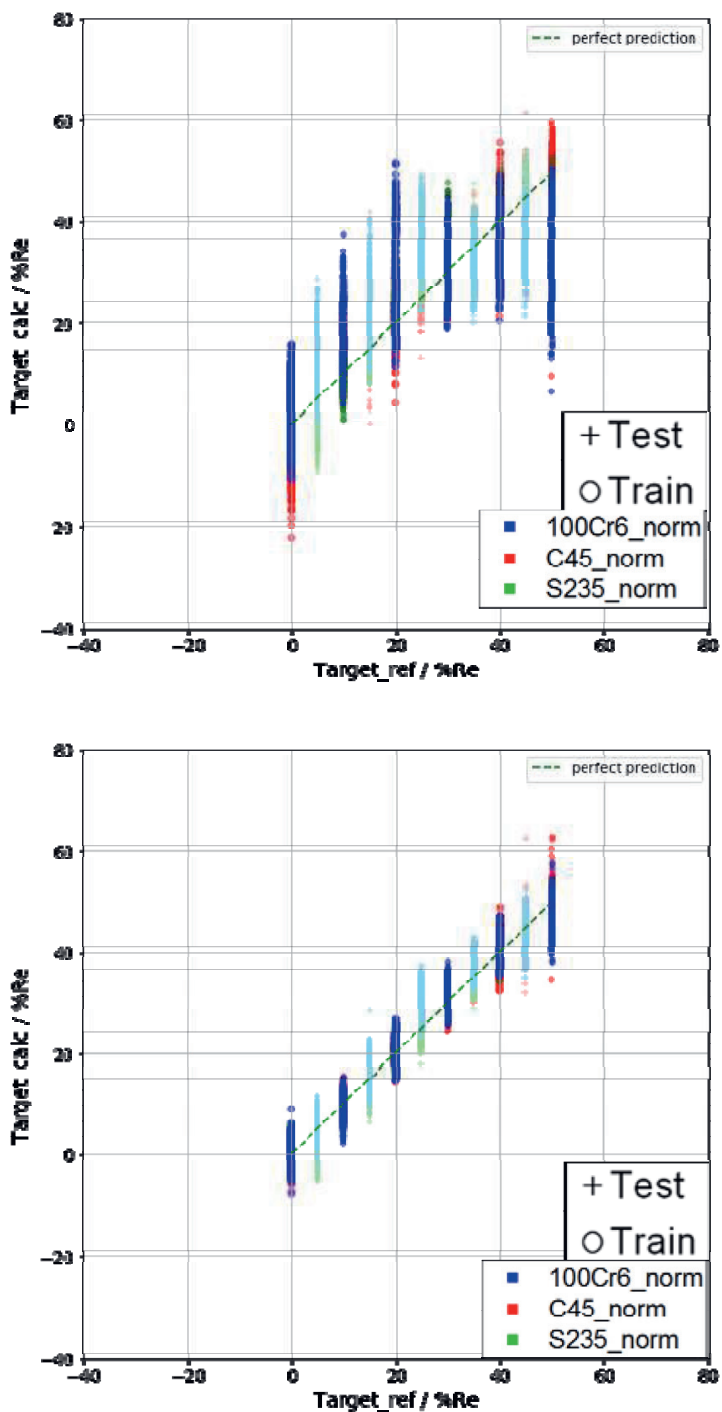

Abb.4: $\quad$ ML-R Abbildung der Trainings- und Testdaten des subjektiv (oben) und automatisiert extrahierten (unten) Merkmalsraum

Im Vergleich beider Merkmalsräume (vgl. Abb. 4) wird eine deutliche Verbesserung der zerstörungsfrei ermittelten Zugspannung sowohl beim Bestimmtheitsmaß $\mathrm{R}^{2}$ Train als auch beim mittleren Fehler RMSETrain (Root Mean Square Error) der Trainingsdaten erreicht. Die Vorhersagequalität der Trainingsdaten verbessert sich von $\mathrm{R}^{2}$ Train $=78,07 \%$ und RMSE $_{\text {Train }}=7,99 \% R_{e}$ auf $R^{2}$ Train $=98,19 \%$, RMSE $_{\text {Train }}=2,3 \% R_{e}$.

Der mittlere Fehler bezogen auf die Testdaten $\mathrm{RMSE}_{\text {Test, }}$ also die Validierung ausschließlich mit Spannungszuständen, die nicht im Training verwendet wurden $(5 \%, 15 \%, 25 \%, 35 \%$ und $45 \% \mathrm{R}_{\mathrm{e}}$ ), zeigt, dass das datenbasierte Modell auf Basis der automatisiert extrahierten Merkmale eine verbesserte Vorhersage des Spannungszustandes von RMSE $_{\text {Test }}=7,83 \%$ $\mathrm{R}_{\mathrm{e}}$ auf $\mathrm{RMSE}_{\mathrm{Test}}=4,99 \% \mathrm{R}_{\mathrm{e}}$ unabhängig vom Werkstoff erreicht. Der Korrelationskoeffzient der Testdaten verbessert sich von $\mathrm{R}^{2}$ Test $=69,35 \%$ auf $\mathrm{R}^{2}$ Test $=87,54 \%$.

\section{Einflussfaktoren auf die Vorhersagequalität datenbasierter Modelle}

Die Vorhersagequalität datenbasierter Modelle hängt von vielen Einflussfaktoren ab. Die im Rahmen dieses Beitrags untersuchten Einflussfaktoren sind die Wahl des Merkmalsraums (subjektiv / automatisiert), sowie die Wahl des Regressionsmodells.

Im weiteren Verlauf dieses Beitrags wird die gleiche Datenbasis wie bei der ML-R verwendet, die Vorhersagequalität der $M L-R$ dient als Referenz bei der Untersuchung der Einflussfaktoren.

\section{Regressionsmodell}

Neben dem Einsatz der multiplen linearen Regressionsanalyse (ML-R) werden im Rahmen dieses Beitrags zwei weitere Regressionsalgorithmen untersucht; die MultiLayer-Perceptron-Regression (MLP-R) [3] und die Support-Vector-Regression (SV-R) [4]. Im Gegensatz zur ML-R besitzen die SV-R und die MLP-R "Stellschrauben" (sog. Hyperparameter), welche einen erheblichen Einfluss auf die Vorhersagequalität der Zielgröße haben. Es gibt verschiedene Ansätze, geeignete Hyperparameter zu finden. Ein einfacher Weg ist die manuelle Optimierung. Hierbei werden die Hyperparameter durch "Ausprobieren" ermittelt, wobei dies bei komplexeren Modellen mit vielen Freiheitsgraden sehr ineffizient werden kann. Durch Einschränkung von Freiheitsgraden kann dieser Aufwand reduziert werden, wobei 
dadurch die Wahrscheinlichkeit, eine (quasi-) optimale Lösungen zu finden, reduziert wird. Im Rahmen dieses Beitrags soll es nicht primär um das Finden eines optimalen Hyperparametersatzes gehen. Es soll vielmehr aufgezeigt werden, ob und mit welchem Aufwand die Vorhersagequalität der ML-R unter ähnlichen Bedingungen (lineare Separierbarkeit) mit komplexeren Algorithmen erreicht werden kann. Um eine Vergleichbarkeit der Regressionsmodelle zu gewährleisten, werden diese im linearen Modus betrieben, das bedeutet lineare Hyperebene bei SV-R und lineare Aktivierungsfunktionen bei MLP-R.

\section{Support-Vector-Regression}

Die Support-Vector-Regression ist ein Regressor aus der Familie der Support-VectorMachine (SVM) [4]. Ziel der SVM ist es, den Bereich um die Klassengrenzen (engl. Margin) frei von Daten fremder Klassen zu halten. Sind die Klassen linear separierbar, wird die Hyperebene ausgewählt, die den kleinsten Abstand zu den Trainingsdaten maximiert. Sind die Klassen nicht linear separierbar, kann durch nichtlineare Kernelfunktionen der zur Verfügung stehende Merkmalsraum in einem höherdimensionalen Merkmalsraum linearisiert abgebildet und nach der Klassifikation wieder in den ursprünglichen Merkmalsraum projiziert werden (Kernel-Trick).

Die Stellgröße bei der Optimierung der Vorhersagequalität im Rahmen dieses Beitrags ist der Parameter $\mathrm{C}$, welcher ein Maß dafür ist, wie scharf die Klassen voneinander getrennt werden (Breite des Margins).

\section{Subjektiv extrahierter Merkmalsraum:}

Um den Einfluss von $\mathrm{C}$ zu untersuchen, wurde dieser im Bereich von $10^{-2}$ bis $10^{4}$ variiert. Abb. 5 zeigt die Abhängigkeit von $\mathrm{R}^{2}$ und RMSE für Trainings- und Testdaten bei Variation von $\mathrm{C}$.
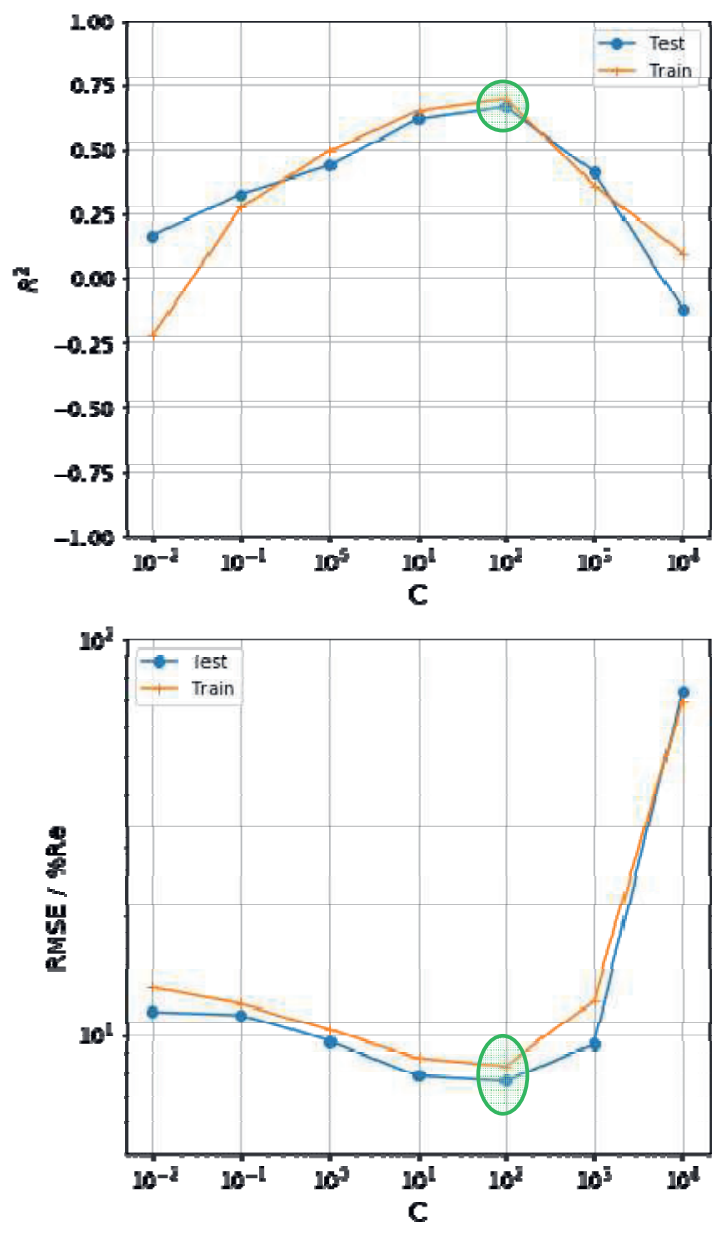

Abb. 5: $R^{2}$ (oben) und RMSE (unten) der Trainings- und Testdaten des subjektiv extrahierten Merkmalsraum für SV-R bei Variation von $C$

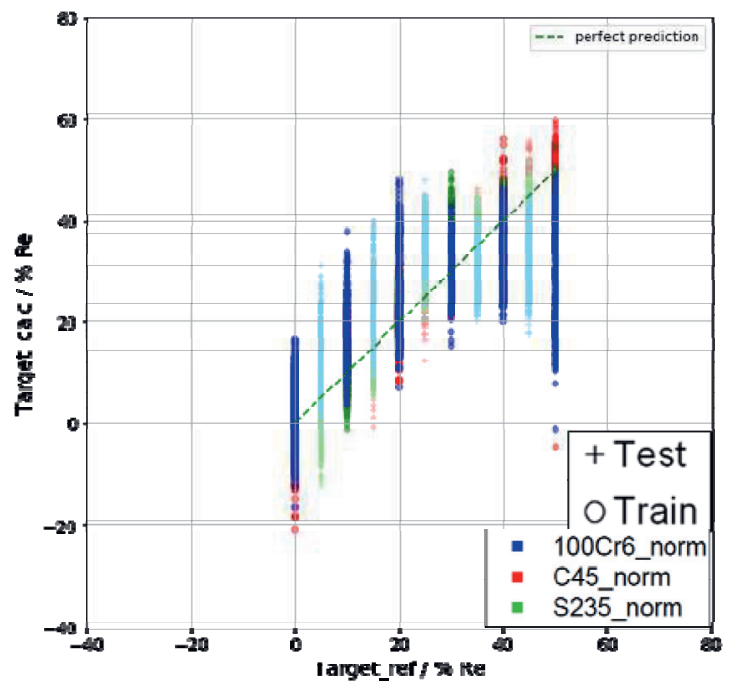

Abb. 6: Abbildung der Trainings- und Testdaten des subjektiv extrahierten Merkmalsraum für SV-R

Das Modell erreicht seine beste Performance von $\mathrm{R}^{2}=69,85 \%$ und ein $\mathrm{RMSE}=8,32 \% \mathrm{R}_{\mathrm{e}}$ 
bei den Trainingsdaten und $\mathrm{R}^{2}=66,69 \%$ und ein RMSEV $=7,62 \% R_{e}$ bei den Testdaten bei $C=100$ (vgl. Abb. 5 und Abb. 6).

\section{Automatisiert extrahierter Merkmalsraum:}

Um den Einfluss von $\mathrm{C}$ zu untersuchen, wurde dieser im Bereich von $10^{-4}$ bis $10^{1}$ variiert. Abb. 7 zeigt wiederum die Abhängigkeit von $\mathrm{R}^{2}$ und RMSE für Trainings- und Testdaten des automatisiert extrahierten Merkmalsraums bei Variation von C. Auch hier hängt die Vorhersagequalität des Modells für die Trainings- und Testdaten signifikant von $\mathrm{C}$ ab, siehe auch Abb. 8.
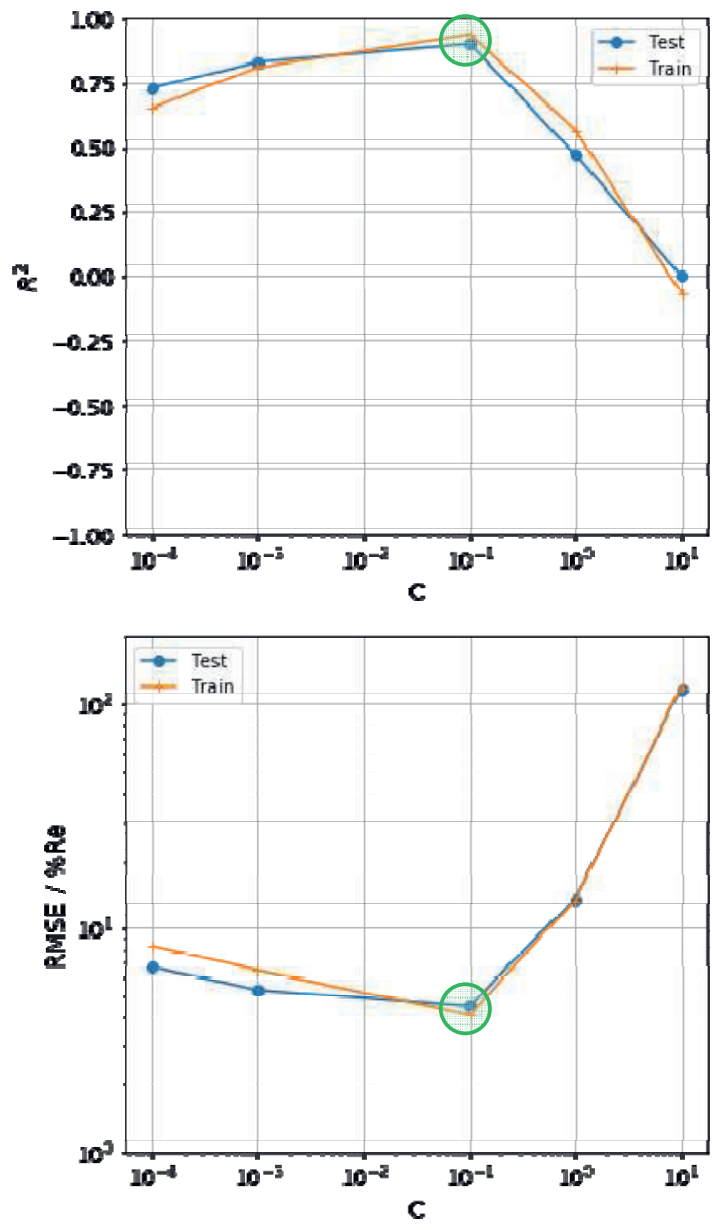

Abb. 7: $R^{2}$ (oben) und RMSE (unten) der Trainings- und Testdaten des automatisiert extrahierten Merkmalsraums für $S V-R$ bei Variation von $C$

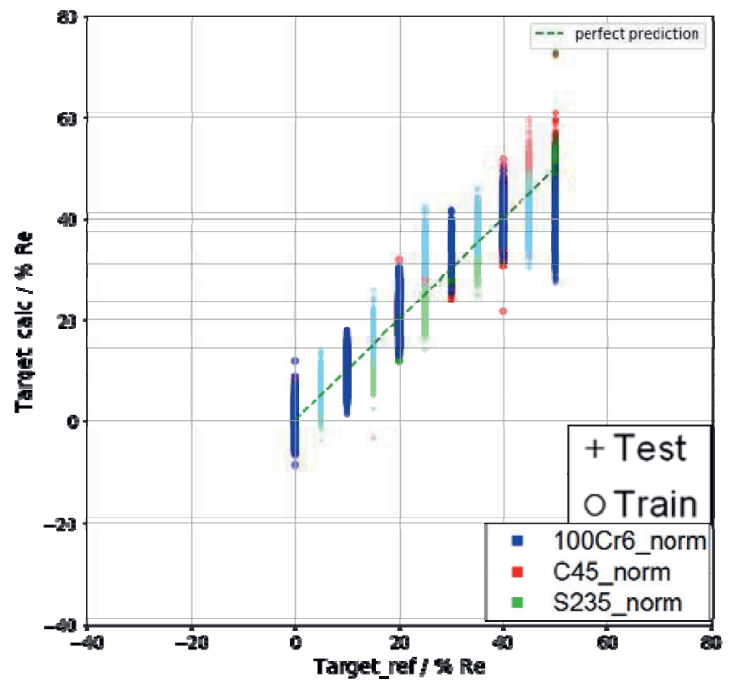

Abb. 8: Abbildung der Trainings- und Testdaten des automatisiert extrahierten Merkmalsraum für SV-R

Das Modell erreicht seine beste Performance mit $\mathrm{R}^{2}=93,96 \%$ und $\mathrm{RMSE}=4,11 \% \mathrm{Re}$ bei den Trainingsdaten und $R^{2}=90,33 \%$ und RMSEV $=4,49 \% R_{e}$ bei den Testdaten bei $\mathrm{C}=0,1$ (vgl. Abb. 7 und Abb. 8).

\section{Multilayer-Perceptron-Regression}

Die Multilayer-Perceptron-Regression ist ein Regressor aus der Familie der künstlichen neuronalen Netze [3]. Es handelt sich hierbei um ein Netz mit mindestens einer verborgenen Schicht (engl. Hidden Layer) von Neuronen. Um den Einfluss der Hyperparameter zu zeigen, werden im Rahmen dieses Beitrags alle Hyperparameter vorgegeben bis auf die Anzahl der Eingangsneuronen im Hidden Layer. Dieser Hyperparameter wird variiert, anschließend wird die Vorhersagequalität der Trainings- und Testdaten bewertet. Die wesentlichen vorgegeben Hyperparameter sind eine lineare Aktivierungsfunktion, ein Hidden Layer, Alpha $=10$ und eine Lernrate von 0,01 .

Lineare Aktivierungsfunktionen finden häufig Einsatz bei Regressionsproblemen. Aus der linearen Aktivierungsfunktion ergibt sich, dass die Neuronen der ersten verborgenen Schicht Linearkombinationen der Eingangsmerkmale darstellen und der Einsatz weiterer Hidden Layer redundant ist, da der Ausgang jeder verborgenen Schicht letztlich wieder eine Linearkombination der Eingangsmerkmale darstellt.

\section{Subjektiv extrahierter Merkmalsraum:}

Um den Einfluss der Anzahl an Neuronen im Hidden Layer zu untersuchen, wurde die Anzahl an Neuronen beginnend mit 1 bis zur 
maximal zur Verfügung stehenden Anzahl an Merkmalen im Merkmalsraum erhöht.
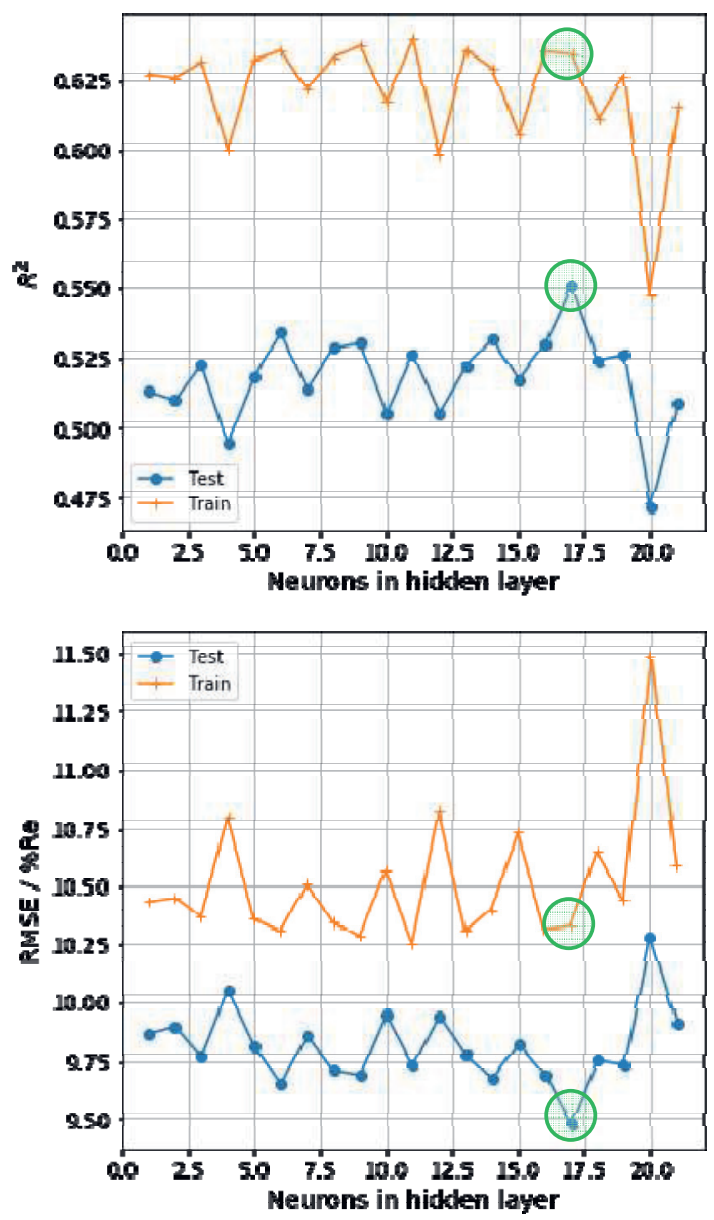

Abb. 9: $R^{2}$ (oben) und RMSE (unten) der Trainings- und Testdaten des subjektiv extrahierten Merkmalsraum für MLP-R unter Variation der Anzahl der Neuronen im Hidden Layer

Es zeigt sich, dass die Anzahl der Neuronen im Hidden Layer in dieser Konfiguration im subjektiven Merkmalsraum keinen signifikanten Einfluss hat. Sowohl die Abbildung der Trainings- als auch der Testdaten verbessert sich nur unwesentlich.

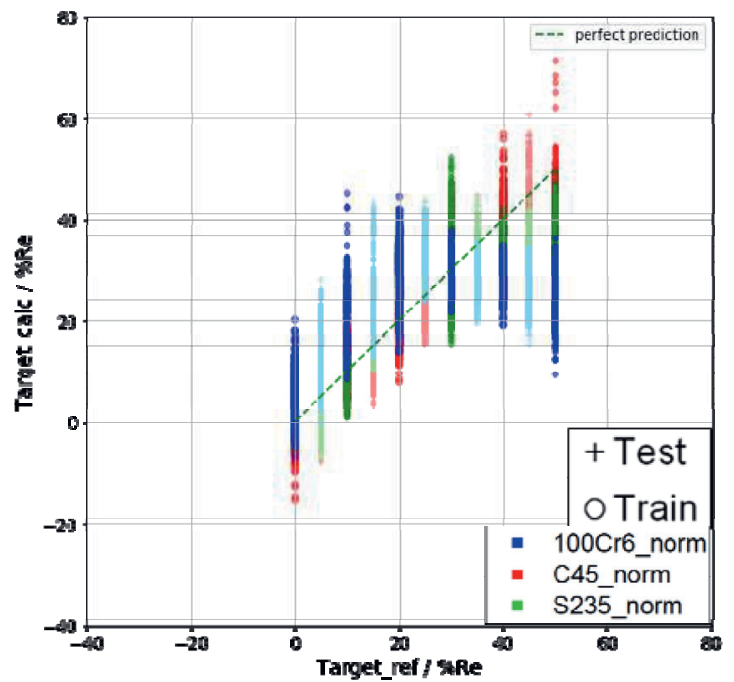

Abb. 10: Abbildung der Trainings- und Testdaten des subjektiv extrahierten Merkmalsraum für MLP-R

Das Modell erreicht seine beste Performance von $R^{2}=63,42 \%$ und ein $\mathrm{RMSE}=10,33 \% \mathrm{R}_{\mathrm{e}}$ bei den Trainingsdaten und $\mathrm{R}^{2}=55,08 \%$ und ein RMSEV $=9,48 \% R_{e}$ bei den Testdaten bei 17 Merkmalen (vgl. Abb. 9 und Abb. 10).

\section{Automatisiert extrahierter Merkmalsraum:}

Um den Einfluss der Anzahl an Neuronen im Hidden Layer zu untersuchen, stehen im automatisierten Merkmalsraum über 270 Merkmale zur Verfügung. Auch im automatisierten Merkmalsraum wurde die Anzahl der Neuronen beginnend mit 1 bis zur maximal zur Verfügung stehenden Anzahl an Merkmalen im Merkmalsraum erhöht. 

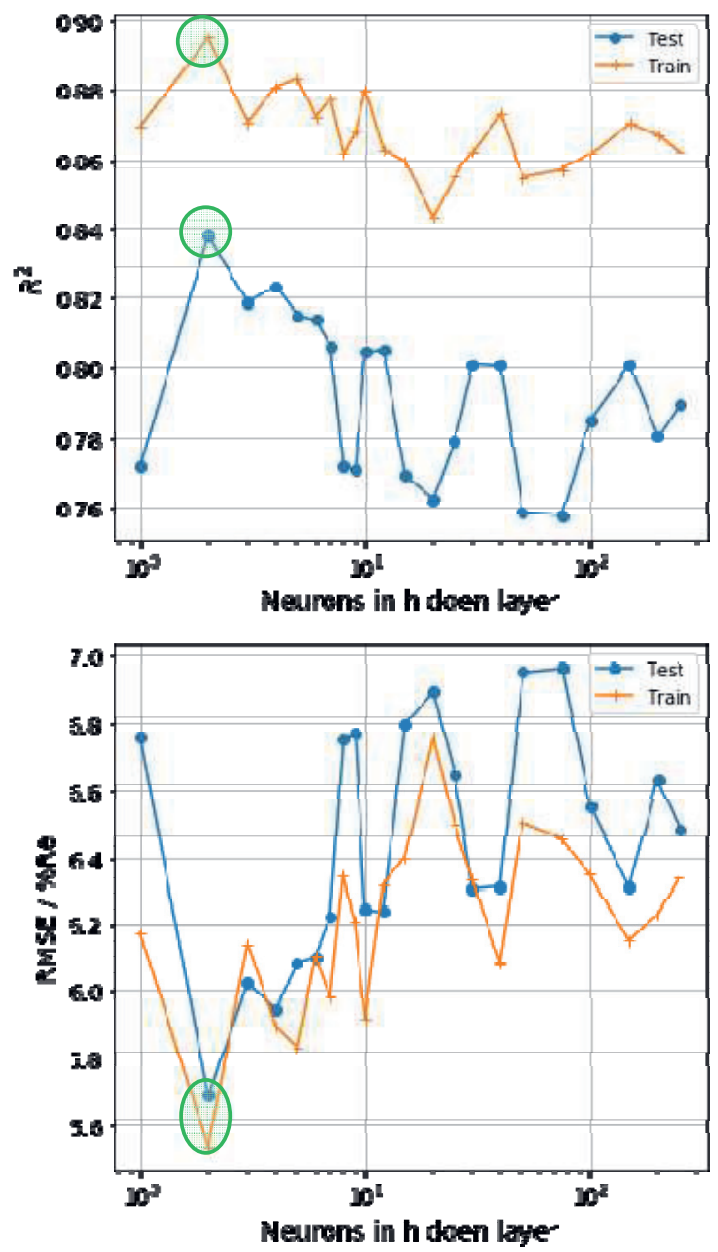

Abb. 11: $R^{2}$ (oben) und RMSE (unten) der Trainings- und Testdaten des automatisiert extrahierten Merkmalsraum für MLP-R unter Variation der Anzahl der Neuronen im Hidden Layer

Es zeigt sich, dass die Anzahl der Neuronen im Hidden Layer in dieser Konfiguration im automatisiert extrahierten Merkmalsraum einen signifikanten Einfluss hat.

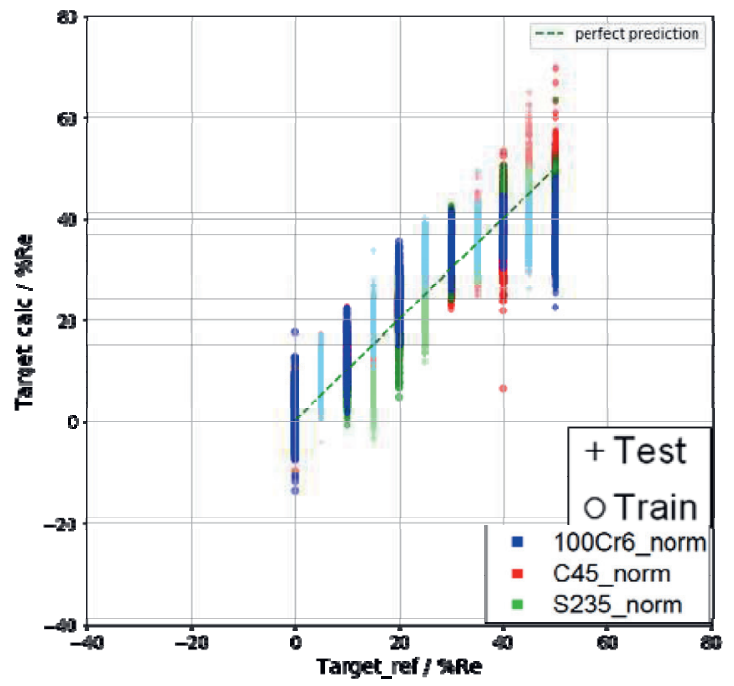

Abb. 12: Abbildung der Trainings- und Testdaten des automatisiert extrahierten Merkmalsraum für MLP-R

Das Modell erreicht seine beste Performance von $\mathrm{R}^{2}=89,49 \%$ und ein $\mathrm{RMSE}=5,54 \% \mathrm{Re}_{\mathrm{e}}$ bei den Trainingsdaten und $\mathrm{R}^{2}=83,82 \%$ und ein RMSEV $=5,69 \% R_{e}$ bei den Testdaten bei lediglich zwei Eingangsneuronen (vgl. Abb. 11 und Abb. 12).

\section{Zusammenfassung und Ausblick}

Im Rahmen dieses Beitrags wurde untersucht, mit welchen Möglichkeiten die datenbasierte Vorhersagequalität verbessert werden kann. Neben der Optimierung des Merkmalsraums wurde die multiple lineare Regression (ML-R) mit weiteren Regressionsalgorithmen, nämlich der Support-Vector-Regression (SV-R) und der Multilayer-Perceptron-Regression (MLP-R), verglichen. Diese Regressoren bieten aufgrund ihrer Mächtigkeit und Flexibilität, Nichtlinearitäten in den Daten abzubilden, enormes Potenzial zur Erstellung komplexer Modelle. Beide besitzen jedoch "Stellschrauben“ (sog. Hyperparameter) die signifikant zur Vorhersagequalität beitragen. Aufgrund der Vielzahl möglicher Kombinationen dieser Hyperparameter kann das Finden einer (quasi-) optimalen Lösung sehr aufwendig sein. Zur Verdeutlichung dieses Aufwandes wurden im Experiment alle Hyperparameter bis auf einen ( $C$ bei SV-R, Anzahl Neuronen im Hidden Layer bei MLP-R) vorgegeben. Dieser diente als Stellgröße und sollte zeigen, welcher Aufwand schon bei der Variation von einzelnen Hyperparametern zum Finden eines (quasi-) optimalen Modells erforderlich sind. Die ML-R wurde als Referenz verwendet. Um eine Vergleichbarkeit der ML-R mit der SV-R bzw. der MLP-R zu erhalten, wurden diese mit linearer Hyperebene im Merkmalsraum bzw. linearer 
Aktivierungsfunktion verwendet. Tab. 1 und Tab. 2 zeigen die modellbasierte Vorhersagequalität der Merkmalsräume sowohl für die Trainings- als auch für die Testdaten.

Tab. 1: $\quad R^{2}$ und RMSE für Trainings- und Testdaten des subjektiv extrahierten Merkmalsraum

\begin{tabular}{|l|r|r|r|}
\hline & \multicolumn{1}{|c|}{ ML-R } & \multicolumn{1}{c|}{ SV-R } & MLP-R \\
\hline $\mathrm{R}^{2}$ Train in \% & 78,07 & 69,85 & 63,42 \\
\hline $\mathrm{RMSE}_{\text {Train }}$ in \% Re & 7,99 & 8,32 & 10,33 \\
\hline $\mathrm{R}^{2}{ }_{\text {Test }}$ in \% & 69,35 & 66,69 & 55,08 \\
\hline $\mathrm{RMSE}_{\text {Test }}$ in \% $\mathrm{Re}_{\mathrm{e}}$ & 7,83 & 7,62 & 9,48 \\
\hline
\end{tabular}

Tab. 2: $\quad R^{2}$ und RMSE für Trainings- und Testdaten des automatisiert extrahierten Merkmalsraum

\begin{tabular}{|c|c|c|c|}
\hline & ML-R & SV-R & MLP-R \\
\hline $\mathrm{R}^{2}$ Train in \% & 98,19 & 93,96 & 89,49 \\
\hline $\mathrm{RMSE}_{\text {Train }}$ in $\% \mathrm{Re}_{\mathrm{e}}$ & 2,3 & 4,11 & 5,54 \\
\hline $\mathrm{R}^{2}$ Test in \% & 87,54 & 90,33 & 83,82 \\
\hline RMSE $_{\text {Test }}$ in \% $\mathrm{R}_{\mathrm{e}}$ & 4,99 & 4,49 & 5,69 \\
\hline
\end{tabular}

Unter diesen Bedingungen ist es also nicht gelungen, eine signifikante Verbesserung der Vorhersagequalität in beiden Merkmalsräumen zu erreichen (vgl. Tab. 1 und Tab. 2). Hierbei ist jedoch anzumerken, dass die wahre Stärke dieser Modelle gerade die Behandlung von Nichtlinearitäten in den Daten ist, die Beschränkung auf eine lineare Hyperebene (SV-R) bzw. eine lineare Aktivierungsfunktion (MLP-R) die Mächtigkeit und Flexibilität dieser Modelle somit stark begrenzt. Neben C bzw. der Anzahl der Neuronen im Hidden Layer gibt es jedoch weitere zu optimierende Hyperparameter. Der Optimierungsaufwand hierfür wächst aber exponentiell mit der Dimension des Hyperparameterraums.

Abschließend lässt sich zusammenfassen, dass bei derart hohem Aufwand für die Optimierung von Hyperparametern die Möglichkeit der Optimierung des Merkmalsraums verbunden mit dem Einsatz linearer Modelle gegenübergestellt werden sollte. Beispielsweise kann die multiple lineare Regression weitestgehend ohne A-Priori-Wissen eingesetzt werden, die Berechnung ist sehr schnell und mit einem aussagekräftigen Merkmalsraum werden sehr gute Vorhersagen erreicht. Im Rahmen dieses Beitrags wurden alle Merkmale der jeweiligen Merkmalsräume ohne Skalierung des Wertebereichs verwendet. Die Skalierung der Daten ist besonders bei der Verwendung von nichtlinearen Aktivierungsfunktionen relevant, da hier stark variierende Wertebereiche in unterschiedlichen Merkmalen das Ergebnis unterschiedlich stark dominieren. Die Wichtigkeit einzelner Merkmale wäre demnach von deren Wertebereich abhängig.
Es ist auch zu beachten, dass für die Zielgröße irrelevante Merkmale die Vorhersagequalität eines Modells verschlechtern können. Eine vorgeschaltete Merkmalsselektion kann die Vorhersagequalität ebenfalls verbessern [5].

\section{Danksagung}

Die im Rahmen dieses Beitrags durchgeführten Untersuchungen wurden mit der Python und der freien Softwarebibliothek ScikitLearn durchgeführt [6]. Ein besonderer Dank geht an die gesamte Community, die es uns Anwendern ermöglicht, die zur Verfügung stehende Infrastruktur und Tools einzusetzen.

\section{Literaturnachweis}

[1] Kneller, E.: Ferromagnetismus. Springer-Verlag Berlin, Göttingen, Heidelberg. 1962.

[2] Youssef, S.; Zimmer, C.; Szielasko, K.; Schütze, A.: "Bewertung subjektiver und automatisierter Merkmalsextraktion periodischer Zeitsignale am Beispiel des 3MA-X8Verfahrens" tm - Technisches Messen, 0.0 (2019): Retrieved 24 Jan. 2019, from doi:10.1515/teme-2018-0074

[3] Robinson, R.: Neural networks offer an alternative to traditional regression Geobyte, February (1991), pp. 14-19

[4] Drucker, H.; Burges, C.; Kaufman, L.; Smola, A.; Vapnik, V. 1997: "Support Vector Regression Machines", in Advances in Neural Information Processing Systems 9, NIPS 1996, 155-161, MIT Press.

[5] Schneider, T; Helwig, N and Schütze, A: Automatic feature extraction and selection for classification of cyclical time series data, tm Technisches Messen (2017), 84(3), 198-206, doi: 10.1515/teme-2016-0072

[6] Pedregosa, F. et al.:Scikit-learn: Machine Learning in Python, JMLR 12, pp. 2825-2830, 2011. 